\title{
Integration of Cloud Computing with Artificial Intelligence and Its Impact on Telecom Sector-A Case Study
}

\author{
Mounir M. El Khatib1, Ahmed Al-Nakeeb1', Gouher Ahmed ${ }^{2}$ \\ ${ }^{1}$ School of Business and Quality Management, Hamdan Bin Mohammad Smart University, Dubai, UAE \\ ${ }^{2}$ School of Business Management, Skyline University College, Sharjah, UAE \\ Email: m.elkhatib@hbmsu.ac.ae, a.alnakeeb@hbmsu.ac.ae, gouher@usa.net
}

How to cite this paper: El Khatib, M.M., Al-Nakeeb, A. and Ahmed, G. (2019) Integration of Cloud Computing with Artificial Intelligence and Its Impact on Telecom Sector-A Case Study. iBusiness, 11, 1-10. https://doi.org/10.4236/ib.2019.111001

Received: November 13, 2018

Accepted: January 21, 2019

Published: January 24, 2019

Copyright () 2019 by author(s) and Scientific Research Publishing Inc. This work is licensed under the Creative Commons Attribution International License (CC BY 4.0).

http://creativecommons.org/licenses/by/4.0/

\section{c) (i) Open Access}

\begin{abstract}
The technologies cloud computing (CC) and artificial intelligence (AI) are very much on business horizon as smart transformation technologies for companies to be smarter once, so as to render their services in a smart way to their customer, meaning promptly, efficiently and economically that best satisfy the customers. This study is a study of the application of CC, and AI in the leading telecom MGA-MENA Company in the middle-east. The result of the application of the twin-technologies of cloud computing and artificial intelligence is the increase in operational services, product efficiencies and better products and more customer satisfying services of a Smart MGA-MENA Company. The conclusion that follows is that the big telecom enterprises like MGA-MENA with a large customer base and every minute umpteen transactions, cloud computing and artificial intelligence is a new and advanced business opportunity. So, it is suggested that telecoms need to be technologically dynamic and updated.
\end{abstract}

\section{Keywords}

Technology, Telecom Industry, Cloud Computing and Artificial Intelligence

\section{Introduction}

In the present digital transformation age, moving towards cloud computing (CC) has made a massive progress over the past few years, which transforms the traditional network communication architectures into a new one model, aiming to improve agility and reduce operation costs as cloud computing depends on sharing of resources which allow business to introduce new products and servic- 
es in many sectors. Cloud computing can be described as an IT paradigm that enables ubiquitous access to shared pools of configurable system resources and higher-level services that can be rapidly provisioned with minimal management effort, often over the Internet. Due to that, there is a huge amount of data from storage, transactions and connecting devices that become new sources for competitive advantage to support telecom to sustain in highly competitive environment. This means telecom needs to be solution and service oriented and only connectivity provider. For example, an MGA-MENA Company, a world-renowned telecommunications group, currently operating in 16 countries across the Middle East, Asia, and Africa, has deployed its cloud computing models offering significant improvements in its services and it aims to be the smart company making use of artificial intelligence in running its operation and managing customer experience.

Today, telecommunication companies like MGA-MENA operate in complex environments and depend on the technology to run and develop scalable network and deliver services to customers, where the main challenges for firms are to sustain by innovation and to improve, which is the only way forward. They have to make an intelligent and economical use of the new technologies of cloud computing and artificial intelligence, which are advancements or a step or two forward over computing and human intelligence. Robotics is another field of technological advancement. Beautifully, they are not competitive but complementary "techs".

In this new business scenario of giant organizations and new technologies, telecoms stand out with their huge geographical and customer reach and number and complexities of their operations. The MGA-MENA, one of the largest telecom companies, has its operations spread over in the Middle East, Asia and Africa, with a big scale of operations.

The objective of this study is to find:

1) How integration between cloud computing and artificial intelligence (AI) will benefit and transform the operation in telecom sector.

2) How AI impacts the operations in MGA-MENA with few successes or planned approaches along with their impacts on operational excellence or customer experience that support to keep its position and rank higher as ICT in the MENA region.

Artificial intelligence is known to be a big technical thing today, which is widely spreading its use and applications. It is the opposite of HI. It is said that artificial intelligence succeeds where human intelligence fails.

\section{Literature Review}

To start with, [1] in a seminal study of cloud computing vis-à-vis telecommunication companies brings out benefits out many benefits of cloud computing that accrue telecommunications in terms of capital, cost, time, marketing, customer relations, delivery of service, customer satisfaction, and not to say of profits. 
They are free from computing problems, which are only means to business ends, can concentrate in service delivery to the best satisfaction of the customers. In sum, cloud computing is a better means of realizing business goals.

In another study [2] suggests that the traditional strategy approach is increasingly ill equipped for a business environment that is being radically changed by new digital technology. The strategy development approaches need to be revised to remain relevant in the world of artificial intelligence, digital reengineering and cognitive computing. Dubai e-government is successful because of excellent information technology infrastructure, government support for innovations, sufficient finances, and the high e-participation of individuals and corporations [3].

Artificial intelligence is said to play an important role for telecom companies as it is customer profiles and provide them with offers based on their needs and interest. Moreover, AI supports operation team, by detecting and predicting system failure, and thereby provides instant corrective action [4]. Furthermore, AI can tackle customer service, for example, TOBi is a chatbot introduced by Vodafone to help customers by answering enquires online, support for problem trouble shooting, that will increase customer satisfaction [5].

According to [6], there has been an increase in the number of users of CC and $\mathrm{AI}$ and also the growth in telecommunication network size. The industry has experienced different needs and preferences from various users. The demand for customized networks has increased and this has made it impossible for this industry to manage their operations without artificial intelligent (AI). Cloud robotics is a rapidly developing technology made possible by the current ubiquitous internet connectivity and the growing number of powerful cloud computing services available [7]. The technology has been applied successfully to humanoid, industrial, mobile and other classes of robots, often through direct collaborations between robot manufacturers and major IT companies.

The communication industry is currently struggling with various operational issues such as designing, maintenance and management whereby with AI, industry stands to several benefits. Firms in communication industry need intelligent decisions to manage their complex and dynamic operations. Through deep learning, machines can use existing training data in industry to analyze large amounts of data retrieved through data mining. AI will master this data such as traffic increasing the accuracy of decision made in this industry [6].

AI and cloud computing add value to the telecom sector through increased attention toward security, as there have been increasing incidences of insecurity resulting from broken networks and losses. Using AI, the security of systems has been increased whereby attacks can be detected automatically through machine learning. This has resulted in less attacks adding value to both the users and firms [8].

With the ever-growing size of users in telecom sector, it has become impossible for humans to analyze the large amount of data that can be used in future 
predictions. By AI and cloud computing, it has become possible to analyze large amount of data to classify traffic, have more accurate predictions, and detection of anomalies and thus optimize networks for better performance. This has resulted in a better managed telecom sector that is more efficient, which eventually adds value to the services they offer to users. It is likely that through AI, users in the future will have customized packages and at the same time a specialized network that will meet their needs and this will only be made possible through AI and cloud computing [9].

Nowadays, network is observed by algorithms that look for abnormal build-ups activity which may be a maker of malicious events such as Distributed Denial-of-Service (DDoS) attacks and attempted hacks. AI and CC is faster and more foolproof methods of anticipating threats on the network. [10]. Even though the adoption of $\mathrm{AI}$ in cloud is inescapable, there are challenges associated with it, and the significant one is to equip Manpower with skillsets on AI with cloud computing. The telecom sectors which apply that system will have to be prepared to spend a substantial amount of money on their staff to support them with knowledge and right skills to enable their success [11].

Another challenge is investigating AI technology to perform predictive analytics. They are using cloud services to build and train machine learning models that help them to gain valuable, actionable insights. But these models are helpful only if they are supplied with the significant amounts of data. So, before deploying a cloud-based AI service, sectors must be sure that they have sufficient security in place to protect their private data and that they are meeting all their compliance requirements [12].

According to a survey Accenture undertook to measure artificial intelligence potential economic impact on 16 industries by [13] found that AI can augment labor productivity by taking on low value-added or supporting tasks and thus enable workers to focus on high value work and businesses in every industry needs to consider $\mathrm{AI}$ as a potential change agent in their investment, innovation and human capital development strategies.

According to [14] the artificial intelligence-based technologies call for alterations in several branches of law; while interface technologies show the difficulty and complexity of regulating interdisciplinary fields.

In the recent times, cloud computing and artificial intelligence technologies are introduced to various telecom sectors to improve performance, productivity and to increase satisfaction. Adopting these CC and AI technologies in telecom sectors poses a huge challenge in training and implementation [15]. Nonetheless, using CC and AI will improve several areas performance and productivity, reduce costs, improve sustainability, and increase users' satisfaction, retention and loyalty in the long run. However, new threats may have to be taken care, for example, security, privacy, legal conflicts and risky reputation if it is used inappropriately. 


\section{Data Collection and Methodology}

The methodology used for the study of the application of CC, and AI in the leading telecom MGA-MENA Company in the middle-east is mainly through the primary and secondary method, which includes, interviews, observation, discussion with staff or groups and document in Engineering department which turned out to be good sources of information on the topic of the study. It was clearly observed that telecoms are playing a major role in artificial intelligence revolution, MGA-MENA Company had adopted this approach to eliminate human errors, and move towards operation excellence, reducing cost and faster time to respond to failure in network is the result of all this is to better customer experience.

Artificial intelligence and cloud computing had put MGA-MENA as the leader in ICT domain in the United Arab Emirates. Cloud computing had enabled the MGA-MENA Engineering to have centralized database of all records of the networks, customer connectivity and data traffic. This allowed the Engineering to develop from a reactive to a proactive company where AI plays a major role which is expected to be increased gradually in coming years. Further, MGA-MENA Company is working to build two state of art Point of Presence POP to service EXPO2020 in Dubai that is fully cloud based with correlation with artificial intelligence that represent future telecom network, to deliver a cutting-edge digital experience to 25 million expected visitors.

\section{Digital Transformation Strategy}

MGA-MENA Company has a clear journey roadmap to fulfill the digital transformation strategy driven by AI. Hence, the telecom network was transformed from legacy architecture to the telecom cloud architecture; with the deployment of data centers, network function virtualization, NFV \& Software-defined networking SDN touching the way of storing data, network itself and infrastructure topology, to provide intelligent and faster service to customer, improve operation practice and enable quick deployment of network technologies, as many current and future demand required features or capabilities that old telecom network cannot provide or have limitation for implementing comprehensive solution driven by AI [16]. This transformed Engineering and IT infrastructure is simpler with less hardware, and is more agile and flexible in term of vendor selection and separated hardware than software, which was one of legacy network limitation. This means the telecom becoming more software-based with centralized controlling (called orchestrator in telecom) which acts as brain which benefits from modern Application Programming Interface API which support MGA-MENA to shift responsibility of service resiliency from hardware to software. Therefore, it is essential to guarantee that awarded vendor is adhering to standards of Cloud Intelligence architecture principles and supports MGA-MENA Company.

For examples, telecom equipment NMS is being shifted to be software in ra- 
ther than hardware, and some Mobile Core system such as Evolved Packet Core EPC become virtual over cloud network benefiting from NFV infrastructure and open door for intelligent operation supported by AI and machine learning enabling Engineering in MGA-MENA to have high optimization, efficient resource utilization and reduced CAPX cost.

It is worth to mention that MGA-MENA shifted their access network to fiber topology to become more agile via Gigabit Passive Optical Network (GPON) where penetration exceeded $92 \%$ in populated area and presently network will be further enhanced with Virtual customer premises equipment (vCPE) that enable moving some of router function to cloud, which will support MGA-MENA Company to simplify and speed service delivery and open door for innovative service or solution and this mainly targets enterprise as phase one, where it will enable MGA-MENA Company to implement AI or machine learning at the top of this solution to enhance customer experience, deliver new product faster and improve service management [17]. Another transformation is moving mobile access network from silo site structure to cloud/virtual Radio Access Network vRAN by pooling Baseband Units BBU's in central exchanges and front haul connectivity will be from POP to last mile to provide better features to customer, optimize network and enable intelligent service provision to customer in term of bandwidth requirement, traffic streaming and mobility connectivity.

\section{Operation Excellence}

The correlation of cloud with AI allows Engineering to benefit from cloud services with NFV to capture all networks' events and start machine learning powered by integration with orchestrator based on APIs and multiple sources of database, virtual network and SDN which would lead to continuously improved customer experience and provide innovative self-care portal to customer and keep network in optimum level by adopting self-healing. Also, by having NFV network powered by artificial intelligence, automated network optimization can be achieved benefiting from the data analysis done by AI engine from multiple sources and knowledge database along with machine learning leading to better support for traffic optimization, resources relocation, predicting failure and doing continuous assurance for network. Due to that operation staff now free of many repetitive tasks can focus on area that need their expertise, which support MGA-MENA to reduce OPEX, better respond to network issue and auto fault restoration. However, the usage rate is still low and the target to reach more than $20 \%$ of operation activities to be done via AI, which is only possible for service or network on Cloud/NFV.

Three examples of the benefit explored in MGA-MENA are self-service portal (Motiv), auto self-healing and auto capacity planning in access network side. Starting with Motiv, customer can troubleshoot his e-Life line services via MGA-MENA App where system will do full analysis of customer problem and then do necessary change and rectification without call and contacting MGA-MENA 
Company. Such solution support to resolve more than 1 million cases, and such solution was not possible over legacy network. Second, network self-healing where millions of data including network behavior, network traffic and NMS alarms indication are continuously monitored and analyzed to predict any possible failure with end to end performance evaluation, where AI does comprehensive verification of network, including checking if there is planned outage in network to do best re-route of traffic and allocate required resources.

It is important to mention that in all cases ticket is open to capture all data related to incident or performance issue, which is fuel for machine learning. However, adaptation of this is still at the beginning and not at expected level. There is a long future for network self-healing.

Third, auto capacity planning, which is still under developed and is currently done manually by analyzing data from different sources such as CRM, system connection inventory and forecast. Then plan is set for access network system expansion yearly and recently every 6 months, it is a lengthy process and needs expert planning. However, AI will be doing frequent data analysis from all sources to build solid plan and reduce error from current practice as system will learn from CRM, velocity of demand on particular area, available resources and it will enrich with updated forecast so plan will be there for right time to do expansion, volume of connection needed, type of customers and load per systems based on requested services to avoid any congestion in system uplinks.

\section{Product Strategy}

MGA-MENA Company has built a system that can learn from customer experience and make on time decision or suggestion for customer requirement via machine learning \& service orchestrator. Also, what supports this is to the MGA-MENA Company strategy to implement vCPE as highlighted earlier as this will lead to revenue generation and customer satisfaction. vCPE will allow targeted business customer only in this stage to select service needed and then process will begin automatically to provision needed services as entire process, system allocation, optimized route and virtual resources will be configured, and service will be ready to use and adjust base on need in short period compared to time taken in traditional lock contracted service where provision of some part is done manually with human intervention. Adding an example for customer experience, is identifying specific type of mobile famous data traffic like Instagram or other social media application and allocate best shortest international route to ensure best on class customer experience.

Another example of how MGA-MENA benefits from the transformation of IPTV under e-life package to NFV \& SDN is where digital store is empowered by AI to bring an exciting and customized experience to each customer depending on AI analytics of data related to customer usage, interest and favorites, as all these data capture enables to recommend and tailor VoD in main screen, also recommend next possible like VoD. Moreover, MGA-MENA Company received 
an award for their innovative e-Life TV interface and changes of how telecom operates such service as engine provider both unique experience per customer and presents in a way that attract him based on his profile, also and differentiation is there for adult or kids.

\section{Use of Case Support and SME}

To increase the benefit of this correlation with engaging staff is to be part of this transformation, MGA-MENA Company established Lab with an environment similar to its network with variety of functions and tools, so staff can test and verify needed approach. For received ideas expert team will work to examine the concept to maximize the number of use cases and benefit from SME in team domain to transfer work toward AI Cloud.

\section{Analysis}

The qualitative data was analyzed based on key work related to concept and then coding was done to identify the benefit of cloud computing with artificial intelligence correlation where cloud computing, and AI are inter-related technologies for telecom world that support them to be more agile, deploy service faster and be smarter operation. With this correlation, it was clearly observed that service provider can continuously improve customer experience in an autonomous way as well as support in auto network optimization, self-healing and intelligent capacity planning. The correlation benefits telecom to shift responsibility of service provision from hardware to software, so telecom moves towards software-defined networking along with deploying virtualized network functions powered by artificial intelligence where high computing power supports telecom to develop logic on top of these, which uplift their capabilities that shows direct relationship of the concept that we verified. This is considered major a transformation when compared to traditional telecom network as demand for customized networks has increased and this has made it impossible for the industry to manage its operations without artificial intelligence.

The result of this correlation along with benefits demonstrated in the way MGA-MENA has transformed its infrastructure, doing its operation, deploying service and react to customer experience, also the movement to modernize its network as intelligence cannot be achieved with legacy systems networks. The competitive advantages that MGA-MENA can avail from this correlation is represented in many use cases implemented along with many initiatives in pipe line to fulfill MGA-MENA strategy to be smarter company. The degree of success will depend on several factors appear in concern for security and compliance along with needed regulation to govern this approach in addition efficient storing of data to build a logic for intelligence. Also, telecom needs to take into consideration that machine learning and deep learning techniques require a huge number of calculations to be made very quickly which means they use a lot of processing power so logic/algorithm to be efficient and to use real needed use 
cases to avoid over utilizing the resources from cloud.

\section{Conclusions}

The movement toward cloud computing has made massively progress with most telecom companies aiming to transform legacy network to modernized network dependent on network function virtualization with software-defined networking to compete and sustain in competitive pressure of a fast-changing environment. MGA-MENA Company's many years' working has modernized its network. However, just moving to cloud is not enough, as intelligent decisions to manage complex and dynamic operations become necessity and impossible for humans to analyze a large number of data, where in AI importance comes to add value to cloud leading to better traffic classification, more accurate network fault predictions time optimization and heightened customer services. So, cloud computing and $\mathrm{AI}$ is found to be better business model.

However, the AI and cloud computing model fits well for telecom companies with a big customer base and a large number of operations at any given time. The study is a modest contribution to the knowledge of large business operations like telecoms and how they can make themselves there effective by not only management but also technological innovations such as the integration of cloud computing with artificial intelligence.

\section{Acknowledgements}

We would like to express our sincere appreciation to all those who provided us with needed help and facilitated to the success of this research study through different forms of contribution especially staff in Technology Department in MGA-MENA.

\section{Note}

The original name of the company is replaced with the MGA-MENA Company for confidentiality.

\section{Conflicts of Interest}

The authors declare no conflicts of interest regarding the publication of this paper.

\section{References}

[1] Joshipura, A. (2018) Bringing Benefits of Cloud Computing to the Telecom Market. https://telecom.cioreview.com/cxoinsight/bringing-benefits-of-cloud-computing-to -the-telecom-market-nid-2931-cid-39.html

[2] Berman, S. and Payne, P. (2018) The Interaction of Strategy and Technology in an Era of Business Re-Invention. Strategy \& Leadership, 46, 10-15. https://doi.org/10.1108/SL-10-2017-0096

[3] AlSayegh, A., Hossan, C. and Slade, B. (Forthcoming) Radical Improvement of 
E-Government Services in Dubai. International Journal of Services Technology and Management. https://doi.org/10.1504/IJSTM.2019.10017173

[4] Nigam, N. (2018) Ways in Which Telecom Companies Drive Competitive Advantage Using AI.

https://telecom.economictimes.indiatimes.com/tele-talk/ways-in-which-telecom-co mpanies-drive-competitive-advantage-using-ai/2797

[5] Huizenga, E. (2017) Artificial Intelligence in Telecoms-Current State of Play. https://blogs.sas.com/content/hiddeninsights/2017/05/12/artificial-intelligence-in-te lecoms/

[6] Kumar, M. (2016) An Incorporation of Artificial Intelligence Capabilities in Cloud Computing. International Journal of Engineering and Computer Science, 5, 19070-19073. https://doi.org/10.18535/ijecs/v5i11.63

[7] Bogue, R. (2017) Cloud Robotics: A Review of Technologies, Developments and Applications. Industrial Robot: An International Journal, 4, 1-5. https://doi.org/10.1108/IR-10-2016-0265

[8] Otani, T., Toube, H., Kimura, T. and Furutani, M. (2017) Application of AI to Mobile Network Operation. ITU Journal: ICT Discoveries, Special Issue, 1, 1-7.

[9] Guibao, X. (2016) A Technological Architecture of Artificial Intelligence. Telecommunication Network Technology Journal, 12, 1-6.

[10] Eden, D. (2017) How AI Will Transform Networking.

https://www.networkcomputing.com/network-security/how-ai-will-transform-netw orking/401941015

[11] Gaurav, S. (2018) Top Cloud and AI Trends for 2018. https://dzone.com/articles/top-cloud-and-ai-trends-for-2018

[12] Harvey, C. (2018) AI in the Cloud Boosts Cloud Performance. https://www.datamation.com/cloud-computing/ai-in-the-cloud-boosts-cloud-perfo rmance.html

[13] Plastino, E. and Purdy, M. (2018) Game Changing Value from Artificial Intelligence: Eight Strategies. Strategy \& Leadership, 46, 16-22. https://doi.org/10.1108/SL-11-2017-0106

[14] Szekely, I., Szabo, M. and Vissy, B. (2011) Regulating the Future? Law, Ethics, and Emerging Technologies. Journal of Information, Communication and Ethics in Society, 9, 180-194. https://doi.org/10.1108/14779961111167658

[15] Issa, T., Isaias, P. and Kommers, P. (2016) Editorial: The Impact of Smart Technology on Users and Society. Journal of Information, Communication and Ethics in Society, 14, 310-312. https://doi.org/10.1108/JICES-09-2016-0035

[16] Lanner (2018) 4 Edge Computing Technologies Enabling IoT-Ready Network Infrastructure.

https://www.lanner-america.com/blog/4-edge-computing-technologies-enabling-iot -ready-network-infrastructure/

[17] Rasheed, A. (2017) Etisalat Digital: From Connectivity Provider to Digital Player. https://gulfnews.com/business/sectors/telecoms/etisalat-digital-from-connectivity-p rovider-to-digital-player-1.2101921 